\title{
LA RECESION DEL NEOCORPORATISMO EN ESPAÑA
}

\author{
Carlota Solé \\ (Universitat Autònoma de Barcelona)
}

\section{ETAPAS EN EL DESARROLLO DEL CORPORATISMO *}

No resulta descabellado afirmar que en España se han sucedido tres momentos y tres tipos de neocorporatismo, en su historia más reciente. El régimen franquista avaló un rígido corporatismo de Estado que esta. bleció la inevitable obligatoriedad de afliarse a instituciones como los Sindicatos Verticales cualquiera que fuere la actividad desempeñada, se fuera empresario u obrero. La ideología que sustentaba la idea de que toda persona económicamente activa era defrnible como productor no pudo contratrestar la fuetza de la realidad de la negociación, encubierta pero efec-

* Actualmente se acepta plenamente el anglicismo "corporatismo" en ia literatura sociológica sobre este fenómeno social. La distinción semántica de este término con el antiguo acorporativismon, que tiene connotaciones fascistas o medievales, según el contexto, responde a la necesaria clarificación conceptual al referimos a un fenómeno social nuevo y reciente en la historia de las sociedades avanzadas de corte occidental.

1. La defnicion de nexcorporativismo que preside nuestro razonamiento es la de Schmitter, $\mathrm{Ph}_{1 .:}$ «Un sisterna de representación de intereses, en el que las unidades constituyentes se organizan en categorías, limitadas en número, específicas, obligatorias, no competitivas, ordenadas jetárquicamente y diferenciadas funcionalmente; categorías reconocidas o permitidas (si no creadas) por el Estado y a las que se garantiza, deliberadamente, un monopolio de tepresentación a cambio de observar algún tipo de control sobre la selección de los lideres y la articulación de derzandas y apoyos» (Schmitter, $\mathrm{Ph}$, en Schmitter, Ph. y Lehmbruch, G. (1979) (eds.), Trends Towards Corporatist Intermediation, Contemporary Political Sociology, vol, 1, SAGE Publications, Londres. Beverly). 
tiva, sobre los salarios y las condiciones de trabajo entre los empresarios y los obreros, a pesar de que el Gobierno las impusiera directamente en los últimos años del franquismo.

Los cargos de representación de empresarios y trabajadores en los Sindicatos Verticales eran nombrados por el Gobierno y debían ser miembros de la otganización política de la Falange, única permitida bajo el régimen franquista. De ahí que el control del Gobierno sobre el sindicato obligara a los empresarios a buscar canales alternativos de presión a través de las Cámatas de Comercio e Indistrial, por ejemplo; y que los empresarios intentaran vías alternativas de negociación del conflicto laboral a través de contactos «clandestinos» con los movimientos sociopolíticos organiza. dos de la clase obrera.

Paulatinamente, a partir de 1958 y hasta 1973 aproximadamente, las razones económicas privan sobre las ideológico-políticas. Como afirman Martínez Alier y Roca Jusmet: «La legislación sobre convenios colectivos de 1958 y de 1973 declaraba como objetivo de la negociación colectiva la integración en una comunidad de intereses y de propósitos de los distintos elementos personales que intervienen en el proceso económico y el fortalecimiento de la paz social. Pero, al mismo tiempo, hubo un énfasis nuevo sobre el hecho de que dicha negociación contribuiría a aumentar la productividad. El período de supremacia de la economía estaba justo empezando cuando el régimen franquista aún había de durar casi veinte años más. Este período de primacía de la economía aún no ha terminado. La economía había de utilizarse más convincentemente que la vieja ideología corporativista, como una teoría de la armonía social».2 A pesar de la apertura económica, el controi del Gobierno sobre los salarios fue más fuerte que la pretendida descentralización en la firma de convenios colectivos o la imagen aparentemente liberal que el régimen intentaba dar.

La economía sigue primando en las negociaciones a espaldas de los gobiernos en muchas empresas, a partir de consolidarse el fuerte crecimiento industrial en la década de los años sesenta, por encima de la polí. tica encorsetada a nivel sindical a través de las organizaciones verticales. El sindicalismo se desarrolla en forma de movimiento sociopolítico do. tando a los trabajadores de auténticos representantes en el centro de trabajo, de la mano de Comisiones Obreras. La realidad se impuso una vez más y los Sindicatos Verticales se mostraron muy pronto incapaces de evitar el arbitraje de obligado cumplimiento en la negociación colectiva y fracasaron en establecer normas para controlar los salarios. De acuerdo

2. Martinez Alier, J. y Roca Jusmet, J, «Economía política del corporativismo en España, del franquismo al posfranquismo, Papers, núm. 24, Barcelona, 1985. 
con ese análisis, el primer momento y tipo de corporatismo en la época franquista es débil, más que suave, ${ }^{3}$ por cuanto los acuerdos tripartitos entre asociaciones empresariales, sindicatos y el Estado (a través de los gobiernos) no se producían, no obstante la ideología corporativista dominante de aquel tégimen politico.

Un segundo momento o tipo de corporatismo corresponde a la época de la transición democrática que se inicia a la muerte de Francisco Franco y el nombramiento de Adolfo Suárez como primer mandatario español. Se hace preciso, hacia 1977 , hallar interlocutores válidos. Los sindicatos son sensibles a esa nueva realidad y moderan sus manifestaciones verbales y posturas radicales en beneficio de un potencial entendimiento con la patronal y el Gobierno. Permiten que los partidos políticos tomen el protagonismo que a ellos atañe para establecer el primer pacto, más político que político-económico, entre los agentes sociales decisivos en aquel momento. Al Pacto de la Moncloa 1977 le siguen ottos pactos sociales en $1979,1981,1982,1983$ y 1985, en los que se toman medidas concretas de concertación social sobre los salarios, con previsión de aumentos salariales calculados no ya sobre la inflación pasada, sino proporcionales a la inflación prevista.

De esta forma se hace frente a los efectos negativos de la inflación sobre la redistribución de la renta y la asignación de recursos, y se esta blece una guía de la negociación colectiva y de la evolución futura de los precios en la economía. En ese segundo momento de corporatismo español los pactos fueron tripartitos o bipartitos según la coyuntura de las relaciones entre el Gobierno y los sindicatos y los deseos comunes de consolidar la democracia y aplacar los ánimos del Ejército. El Acuerdo Marco Interconfederal, el Acuerdo Nacional sobre el Empleo, el Acuerdo Interconfederal y el Acuerdo Económico y Social fueron pactos corporativos por el contenido y naturaleza de las medidas pactadas y las consecuencias en la disminución de la conflictividad laboral resultantes. Fueron pactos corporativos más allá de la concertación social porque imprimieron a las relaciones industriales la impronta de la incipiente cultura económica basada en la racionalización de la puesta en juego de intereses contrapuestos. De ahí que pueda afirmarse que nuestro país ha transcurrido por la senda del corporatismo a semejanza de otros países occidentales, a pesar de quedar tantas veces reducidos los acuerdos a las cuestiones salariales, olvidando otros temas cruciales en un marco de relaciones industriales, de haber perdido tantos afiliados los sindicatos mayoritarios y haber crecido la impresión entre Ia opinión pública de que sólo una minoría (de un diez

3. Maraffi, M. (1982), La società neocorporativa, Il Mulino, Milán. 
a un veinte por ciento) de los trabajadores asalariados estaban tealmente representados en las negociaciones.

Cabría preguntarse si el tercer momento del transcurso corporativo en España se inicia por la inflexión aparentemente irreversible en el clima de consenso y negociación de la concertación, o bien significa un paso más en la misma dirección. El crecimiento económico a partir de la segunda mitad de los años ochenta aproximadamente, ha coincidido con un período de estancamiento e incluso de disminución real de los salarios. Esa coincidencia, insostenible desde la perspectiva sindical, llevó a la jornada de huelga general del 14 de diciembre de 1988. Inmediatamente, los sindicatos recuperan viejas expresiones y consignas, antiguas manifestaciones y actitudes. Los conflictos sectoriales y las huelgas puntuales se suceden de manera intermitente avalando la correlación entre la inexistencia de acuerdos corporativos y el aumento de la conflictividad laboral.

El núcleo de la cuestión está en si hemos entrado en un nuevo momento o período, cualitativamente distinto de los dos anteriores y duradero en el tiempo, en la vía de la consolidación del corporatismo, o bien ha concluido el hechizo de la concertación social y su potencial encantamiento de las relaciones entre los agentes sociales, una vez desaparecidas las condiciones políticas y económicas que la habían hecho necesaria. La alternativa de la negociación libre de los salarios y condiciones laborales podría flexibilizar la estructura de salarios adaptándola a las condiciones de productividad y rentabilidad de cada empresa pero, a corto plazo, podría crear tensiones en el metcado de trabajo, especialmente ante el reto de la aplicación del Acta Única Eutopea después de 1992.

Naturalmente, las razones de competitividad y de aumento de la productividad influirían la determinación de los salarios en un matco de negociación libre. Ello supondría un nuevo aprendizaje e internalización de valores sobre el trabajo y el empleo que requeriría un espacio de tiempo dilatado y no evitaría los conflictos puntuales en muchos sectores productivos. La negociación debería establecerse sobre presupuestos nuevos, en todo caso dístintos de los que presidieron los pactos de naturaleza corporativa desde 1977 en España. Los protagonistas podrían variar en relevancia por cuanto los gobiernos dejaxían de controlar la negociación centralizada de las bandas salariales y actuarían con menor contundencia en frenar la inflación. Probablemente ello repercutiría en cambiar las pautas de conducta de los agentes sociales en negociar de acuerdo con las previsiones de inflación, orientándose por los indicadores de la evolución de la economía española e internacional.

Esta hipotética situación daría al traste con la práctica de acuerdos corporativos que ha ido forjándose en los últimos diez años a través de 
los avatares y altibajos propios de la implementación de una nueva concepción del trabajo y de las relaciones industriales. Aparte los Pactos de la Moncloa, de carácter más político que económico, los acuerdos firmados desde 1979 supusieron un coste social y bumano que debe rentabilizarse a un plazo mayor que los diez años transcurridos. Por otra parte, la práctica de acuerdos corporativos, expresión de la nueva cultura económica que impregna suavemente las relaciones industriales en España, va pareja a la consolidación de la democracia. Al igual que la transición democrática empieza a tocat fin, comienza a fltrarse una nueva pauta de conducta en relación a la negociación de las relaciones industriales entre sindicatos y asociaciones empresariales. Torcer esa dinámica supondría para los trabajadores, empresarios y Gobietno un coste social aún mayor a medio plazo. Conscientes de nuestro optimismo, intuimos que los agentes sociales son capaces de ponderat los efectos negativos y positivos del abandono total de las prácticas de concertación social y de la tigidez en conceder mecanismos de compensación a quienes conscientemente se avinieron a ceder poder adquisitivo y mejoras inmediatas en pro del bienestar social y la estabilidad política en consonancia con la internacionalización creciente de la economía española.

Ese optimismo se ve enfriado por el análisis de los efectos que han tenido los acuerdos corporativos tras doce años desde la firma de los Pactos de la Moncloa. Uno de los principales objetivos del famoso pacto de 1977 exa la reducción del desempleo, que en lugar de remitir ha seguido creciendo con fluctuaciones estacionales y coyunturales. Tampoco se ha manifestado claramente la relación entre la moderación de los salarios y la tasa de inversión, aumentando los beneficios de actividades especulativas, en comparación con las directamente productivas, de forma asombrosa. El control de la inflación pareció conseguirse gracias a las restricciones de los aumentos salariales en el primer quinquenio de los años ochenta, pero en los últimos dos o tres años la tasa de inflación prevista inicialmente no ha coincidido con la tasa real de final de año. El gobierno socialista ha debido rectificar sus previsiones a la vez que los sindicatos se planteaban la necesidad de revisar su postura ante la moderación salarial asumida sin compensaciones reales.

Por otra parte, los acuerdos corporativos se frmaron en época de crisis económica. Los argumentos políticos y económicos para justificar la política de concertación social se centraban en el mantenimiento de la estabilidad democrática y en la salida de la crisis gracias al esfuerzo solidario de la población activa, el consentimiento de las clases pasivas en la mejor utilización de los recursos e instrumentos disponibles para relanzar la inversión y aumentar el empleo. Después de un año de elevada 
conflictividad, el pacto corporativo quizá más elaborado y detallado de ese período, el Acuerdo Económico y Social de 1985, hace votos por la mejora del clima de confianza necesario para el desarrollo de la actividad económica, la disminución de incertidumbres, que redundarán en la elevación del bienestar general de la comunidad. ${ }^{4}$

Esos deseos se cumplitán si se resuelve la crítica situación económica fomentando la ocupación a la vez que se mantiene el poder adquisitivo de los salarios, además de hacer frente a la modernización de la economía española ante el reto de la incorporación a la Comunidad Económica Europea. El marco y ámbito en el que debe procederse al saneamiento profundo de la economía, evitando desgastes y conflictos, es el de la negociación a tres bandas, que muy pronto acaba por denominarse concertación social. Envueita en el ropaje de Ia armonía social, la necesidad de corresponsabilidad para una convivencia pacífica y fructífera y la solidaridad de agentes sociales, antagónicos en principio, la concertación social tiene visos de alcanzar los niveles más altos de un acuerdo corporativo en un clima macroeconómico de superación de la crisis y de inicio de una etapa de crecimiento económico.

\section{CRISIS O RECESION DEL NEOCORPORATISMO}

El análisis de las medidas contenidas en el último acuerdo económico y social de naturaleza corporativa es útil para contrastarlo con el momento posterior a la huelga general del 14 de dicicmbre de 1988. El núcleo del AES se centra en los acuerdos sobre materia salarial. Los salarios aumentarán de acuerdo con la inflación prevista, se prevé la posibilidad de no cumplimiento de los incrementos salariales pactados, por parte de las empresas que acrediten, objetiva y fehacientemente, situaciones de déficit o pérdidas mantenidas en los ejercicios contables de los dos años anteriotes a 1985. Para los años posteriores se contempla la previsión de la inflación en la fijación del aumento de salatio. En esos casos deberán reali. zarse auditorías, con la excepción de las empresas de menos de veinticinco trabajadores, para las cuales los informes de los auditores se sustituyen por la documentación en manos de la dirección de la empresa.

Esta posibilidad implica, de hecho, la institucionalización de cierta flexibilidad a la baja que beneficia a las pequeñas y medianas empresas al concretar los salarios. Implica asimismo la renuncia por parte de los sin-

4. Acuerdo Económico y Social, 1985-1986, p. 31. 
dicatos a establecer unos mínimos salariales por sector con la previsible renegociación caso por caso entre las partes afectadas cuando se planteen incrementos salariales menores bajo la justificación de la difícil coyuntura por la que atraviesa la empresa en cuestión. Están también eximidas de acogerse a los incrementos salatiales establecidos las emptesas acogidas a planes de reconversión industrial.

En realidad los incrementos de revisión salarial acaban siendo iguales - inferiores a los incrementos de la inflación prevista, de forma que el poder adquisitivo disminuye sin poder contrartestarse esa disminución en el espacio de tiempo en el que se percibe la cantidad revisada, al ajustarse excesivamente los salatios a la tasa de inflación y no concederse margen alguno de pérdida de poder de compra del dinero. El ajuste en la previsión de la tasa de inflación en los úítimos años por parte del Gobieno avala la pérdida recurrente del poder adquisitivo de los salarios y la tendencia por parte de las empresas a tepercutir sobre los precios la parte de beneficios arrebatada por el salario. ${ }^{5}$

Otros aspectos importantes en relación a las condiciones de trabajo son también contemplados en el Acuerdo Económico y Social, como la seguridad e higiene, el absentismo, derechos laborales, patrimonio sindical y medidas para fomentar la inversión y crear ocupación. En el caso de los cinco primeros temas, las recomendaciones se concretan en medidas antiabsentismo, por ejemplo para controlar el absentismo injustificado y regular el justificado a través de reducciones salariales. Se contempla la posibilidad de introducir nuevas tecnologías, nuevas técnicas de gestión y nuevas formas de utilizar las materias primas por parte de la empresa, previa consulta a los trabajadores, que pueden decidir sobre la salubridad o conveniencia para la salud física y/o mental de ese cambio en el proceso productivo.

Esas breves incursiones de un acuerdo como el AES en la negociación de las condiciones de trabajo abren la puerta tímidamente a la condición de corporatismo de un pacto entre sindicatos, patronal y Gobierno. Las formas de contratación laboral, formación profesional y ocupacional, legislación laboral y otras, atienden tanto a las condiciones de trabajo como a la incorporación al mercado de trabajo de colectivos como los jóvenes, los parados de larga duración y otros. No aportan, por otro lado, ninguna novedad por lo que respecta a los contratos de prácticas, a tiempo par. cial, de relevo o de inicio de una nueva actividad. Obviamente se tegula también el despido.

5. Sampedro, J. L. (1985), La inflacion, la prótesis del sistema, Montesinos, Barcelona, p. 137. 
Aparte los aspectos puntuales y de amplia polémica como el de la transferencial del patrimonio sindical, una parte sustancial del Acuerdo Económico y Social de 1985 se dedica a la negociación de los convenios de centro, a fin de alcanzar como ámbito menor el de la empresa. Los firmantes del pacto se comprometían a alcanzar la unidad sectorial del ámbito nacional, incluyendo en los convenios colectivos las particularida. des del sector y otras cuestiones de orden más general ya establecidas en anteriores acuerdos o pactos de nivel superior. Implícitamente, se daba por supuesto que la negociación se establecería por dos años con los dos grandes sindicatos y en todo el territorio español, para todos los traba. jadores, afiliados o no a las centrales sindicales. Se olvida el constante flujo entre las cúpulas sindicales y sus respectivas bases que podrían controlar el cumplimiento de las medidas contenidas en el acuerdo.

El relanzamiento de la inversión y la creación de nuevos puestos de trabajo fue uno de los objetivos más llamativos del AES. La concatenación del crecimiento inducido de la inversión, a partir de la recuperación de los beneficios empresariales por reducción de los costes salariales y fiscales y en menor grado los financieros, con la creación de empleo es anunciada en el AES como una declaración de política económica por parte del Gobierno. Las medidas para la creación de empleo se concentran en la disminución de la presión fiscal, la lucha contra el fraude y la contención del déficit público para añadir los mecanismos incentivadores de la inversión como la congelación de los tipos impositivos, el incremento de las desgravaciones, las deducciones a la cuota de la Seguridad Social por cada nuevo trabajador ocupado.

Estas medidas fiscales a favor de la inversión no caralizan automáticamente los excedentes empresariales hacia la actividad inversora en actividades directamente productivas. La constatación empirica de que la tasa de paro ha aumentado, a pesar de la disminución de los costes salariales unitarios (y el descenso del poder adquisitivo de los salarios) y la mejora de los excedentes de explotación empresarial, es buena prueba de que no necesariamente aumenta la inversión productiva. Las empresas destinan frecuentemente los beneficios obtenidos en mejorar su propia situación financiera y en algunas circunstancias en tenovar el equipo de capital o modernizar la tecnología. $Y$ ésas son actividades más frecuentes entre las grandes empresas que entre las medianas o pequeñas empresas. En resumen, la creación de empleo no fue el objetivo alcanzado con pleno éxito a través del pacto, pero la coyuntura econónica al término del AES favo. reció indirectamente una mejora en la situación.

La reconversión industrial, sin embargo, se había estado produciendo por sectores, a duras penas y con grandes costes sociales, al margen del 
control directo mediante pactos y acuerdos. Por otro lado, la mejora en la situación económica repercute en un rebrote inflacionista que se manifesta precisamente en el momento en que los sindicatos deciden convocar la huelga genetal del 14 de diciembre de 1988. Aunque los hechos sean económicamente explicables, a nivel social se produce desazón y pérdida de credibilidad respecto a la politica económica gubernamental. Si durante más de un quinquenio esta politica estuvo tespaldada por los sindicatos, con mayor perseverancia acrítica por parte de la Unión General de Trabajadores, a partir de 1987 las denuncias a la quiebra del modelo de concertación social desde el bando sindical son constantes. Se argumenta que los pactos sociales son difícilmente compatibles con medidas de política económica neoliberales, al tiempo que se reta al Gobierno a cumplit su parte en el compromiso del giro social.

Todos esos acontecimientos nos llevan a reflexionar sobre la crisis o incluso defunción, según algunos autores, ${ }^{6}$ de la concertación social, y por ende del neocorporatismo en nuestro país, a la luz de la conceptualización elaborada por diversos estudiosos.' Es evidente que los intentos de definición del neocorporatismo como sistema económico o modo de producción, ${ }^{8}$ como subsistema político, ${ }^{9}$ como estructura política más que ideo logía, ${ }^{10}$ como organizaciones colectivas o colectividades, ${ }^{11}$ como una forma de Estado en el sentido de sistema político global, ${ }^{12}$ como desartollo ultetior del sistema capitalista de producción ${ }^{13}$ o como forma de oposición en las sociedades capitalistas avanzadas a la fuerza de la clase obrera organizada, ${ }^{\text {,4 }}$ no dan cuenta de las dificultades en la puesta en práctica de una política continuada de pactos y acuerdos corporativistas.

6. Segura, J., "Concertación o conflicta", en Santos Juliá (comp.), La desavenencia. Partidos, sindicatos y buelga general, Aguilar - El País, Madrid, p. 279.

7. Sole, C., «El debate corporativismo-neocorporatismo», Revista Española de Investigaciones Sociolbgicas, núm. 26, Madrid, 1984.

8. Winkler, J. T. (1977), «The Corporatist Economy: Theory and Adrninistration», en Scase, R. (ed.) (1977), Industrial Society: Class, cleavage and Control, Geotge Allen an Unwin, Londres.

9. Schmittet, Ph., «Still the Century of Corporatism», The Review of Polizics, vol. 36, núm. 1, 1974.

10. Panitch, L., "The Development of Corporatism in Liberal Democracies», en Schmitter, Ph. y Lehmbruch, G. (eds.), 1979.

11. Offe, C. y Wiesentahl, $H$, "Two logics of collective action, theoretical notes on social classe and organization form Political Power and Social Theory, núm. 1, 1980.

12. Jessop, G., "Corporatismo, Parliamentarism and Social Democracy", en Schmitter, $\mathrm{Ph}$, and Lehmbruch, G. (eds.), 1979.

13. Offe, C. y Wiesentahl, G., op. cil., p. 176.

14. Cawson, A. (1982), Corporatism and Welfare: Social Policy and State Inteyvention in Britain, Heinemann Educational Books, Londres. 
En el caso español, una limitación de implementar esa política ba derivado del papel activo que han jugado los gobienos en la concepción y puesta a punto de los pactos económicos y sociales. Ello ha levantado suspicacias en sus interlocutores, quienes a veces han confundido la actuación gubernamental con la idea de que el estado será activo siempre en la formación del sistema de representación de intereses y en la negociación de pactos y acuerdos de tipo corporativo. Así, se olvida que algunos de esos acuerdos pueden establecerse de forma bipartita entre capital y trabajo, con la indirecta supervisión del Estado que limita conscientemente el alcance de sus propias intervenciones directas.

Además, aun cuando los gobiernos españoles de los últimos doce años decidieran llevar a cabo con éxito una política de rentas, ello no significa que desearan seguir una política intervencionista en lugar de mantenerse en una economía mixta. En varias ocasiones el excesivo margen de maniobra de la autoridad gubernamental no hace más que llenar el vacío de la iniciativa de los interlocutores. No obstante, ésta se desinhibe en cuanto las compensaciones reales como contrapartida a negociat se clarifican.

En la etapa de crisis económica, aproximadamente desde 1975 a 1985, la concertación social se practica por el creciente interés en consensuar mejores condiciones de vida para los trabajadores, a medio plazo; mayor participación en las decisiones de política económica a través de los sindicatos y un más alto nivel de beneficios para el sector productivo de la economía. Debe diferenciarse la concertación social, en el sentido de formulación e implementación de prácticas políticas que configuran un sistema o una estructura dentro del sistema de naturaleza corporativa, de neocorporatismo como sistema de representación de intereses e intermediación de los mismos, ${ }^{15}$ aunque exista empíricamente cierta correlación entre ambos fenómenos en muchos países. ${ }^{16}$ En el caso español sigue manteniéndose la relación estrecha entre grandes organizaciones de intereses y los partidos políticos, además de un nivel elevado de cooperación entre las asociaciones que los representan, que sigue influyendo en el curso de las medidas de política económica, aunque éstas sean dictadas por el Gobierno sin atender a los interlocutores. En ese sentido cabría hablar de recesión coyuntural más que de crisis en la vía del neocorporatismo en España.

Los sindicatos son reacios a defender el modelo retributivo que implican los acuerdos económico-sociales, fundamentados básicamente en la negociación salarial sobre la inflación prevista y en la participación a poste-

15. Schmitter, Ph, op. cit, p. 15.

16. Un ejemplo clásico es el de Austria. 
riori en los resultados de la empresa, porque en realidad la segunda premisa no se ha cumplido y los acuerdos se han reducido a contener los salarios. Los empresatios se han visto atrapados en los primetos años de la negociación a través de pactos por luchar por su supervivencia, olvidándose de prever medidas para garantizar la participación de los trabajadores en los beneficios como resultado de la mejora de la productividad y de la rentabilidad. La etapa de superación de la crisis que se inicia al término de la vigencia del AES coge desprevenidos a los empresatios y pone de manifesto la frustración de los trabajadores. El sistema de telaciones industriales y la política de rentas del gobierno socialista se pone en entredicho, sin conseguir éste salvar la brecha entre la familia socialista y evitar así la quiebra del modelo que el propio Gobierno propugna.

Las razones económicas de esa quiebra se aúnan al entusiasmo de los agentes sociales por haber logrado un clima de entendimiento y colaboración, más allá del contenido económico de los pactos en concreto y sus efectos reales, una vez superadas las reticencias de los empresarios respecto a la política econótnica socialista, la falta de reconocimiento social de los sindicatos y el temor del Gobierno a no conseguir su legitimación ante los poderes económicos privados para llevar a cabo su política de ajustes. ${ }^{17}$

Sin embatgo, cabe preguntarse cómo alcanzar los objetivos de una politica económica que, manteniéndose dentro de márgenes de maniobra aceptables, permita paliar las situaciones de injusticia relativa más flagrantes, mejorar la dotación de bienes colectivos y repartir de forma más equitativa los beneficios de cuatro años de expansión, ya que no se hizo así con los costes de ocho años de crisis. Siguiendo a Segura, puede afirmarse que tanto sindicatos como Gobierno desearían poder generar mucho empleo, proteger a quienes lo precisan y ampliar la oferta de bienes colectivos. Razonabletente, deben establecerse prioridades y valorar el margen de maniobra para obtener esos objetivos de política económica, puesto que difícilmente podrán conseguirse en su totalidad y simultáneamente. Las divergencias surgen entre Gobierno y sindicatos en la estimación del orden de prioridades y la valoración del margen de maniobra.

La creencia del Gobierno en la expansión de la empresa privada como generadora de excedentes y recaudaciones fiscales suficientes para mejorar las funciones públicas de la protección y la provistón de bienes colectivos conlleva estimular el crecimiento económico del sector privado. Para ello, desea mantener los equilibrios macroeconómicos básicos a través del control de la inflación y, por lo tanto, intentando reducit los costes de la produccion. Entre estos costes (importaciones, bienes intermedios, costes finan-

17. Segura, J., op. cit., p. 284. 
cieros, salarios) los más controlables son los salarios y el déficit público. El margen de maniobra es, pues, muy estrecho para el Gobierno. Los sindicatos desean crear empleo arañando los beneficios de las empresas que en los años de reciente recuperación económica han aparecido espectacularmente excesivos. Propugnan aumentar el déficit público y/o la presión fiscal media, y/o la redistribución del gasto público. Por supuesto, no consideran que los salarios causen el aumento de inflación sino el gasto creciente en bienes de lujo. Exigen al Gobierno, en consecuencia, mayor actuación sobre esas variables.

Dadas las diferencias en la apreciación de la situación en cuanto a las causas de la inflación y los efectos del déficit público, las prioridades relativas sobre la composición del gasto público, el grado de confianza en que el sector público pueda actuar en la dirección de los objetivos económicos planteados, etc., ponen sobre el tapete la cuestión del crecimiento del empleo y la remisión del paro. Esos objetivos centrales pueden alcanzarse por un aumento de la producción nacional o por el teparto del empleo existente, a través de la reducción de la jornada media de trabajo, por la reducción de la edad de jubilación, lo cual implica tanto el reparto de las rentas salariales entre ocupados y parados como el aumento de los costes de pensiones y la implementación de un sistema de redistribución de la renta entre los trabajadores de distintas edades.

Tantas dificultades hacen envidiable el aumento del empleo y la remisión del paro a partir del crecimiento económico. Para ello es importante controlar la inflación y los déficit público y comercial. ${ }^{18}$ Deben sin embargo establecerse los límites toletables socialmente y ponderar los efectos en el empleo de medidas económicas antiinflacionistas. De esta forma se respetarían los equilibrios macroeconómicos básicos para hacer frente a la integración completa en la arena económica europea. Los temas de la competitividad de la economía española y el aumento de la productividad van parejos a la atención que merece una política de estabilidad.

Las tazones económicas que avalan la continuidad de una política de concertación social han sido ampliamente discutidas. El trinomio de intereses entre Estado, capital y trabajo se fundamenta en un crecimiento regulado y una concentración económica fuerte, en competencia con otras sociedades occidentales. Los tres protagonistas de los acuerdos y pactos son tacionalmente conscientes del papel que les corresponde en aunar el trinomio de intereses. Las quiebras y recesiones en la vía de la consolidación del neocorporatismo en España no hacen más que mostrar la capacidad de reacción de unos sindicatos más fuertes en su poder de negociación, un

18. Segura, J., ibid., pp. $286-289$. 
Gobierno más flexible con sus interlocutores sindicales a fin de alcanzar el consenso necesario para estar presente, sin fricciones, en la arena internacional, y un empresariado más convencido de compensar con una mayor participación del resto de agentes sociales el incremento de la productividad y la competitividad de la economía española.

La existencia de un orden social estable y pluralista en España garantiza la racionalización del proceso de toma de decisiones por parte del Estado, a fin de incorporar a los grupos de estatus subordinados en el proceso político y en la dirección de ese proceso. Así se logra vincular cohesionadamente la estructura de intereses en el corporatismo societal. ${ }^{19}$ En esa vía se mantiene y preservan las prácticas e instituciones democráticas, el activismo más o menos manifiesto de un sistema de partidos políticos y la existencia de una serie de asociaciones funcionales, estructuradas verticalmente con poder autónomo; el consenso y la persuasión, la atención a la organización de los trabajadores más que la indiferencia a sus reivin" dicaciones. Subyacentes a manifestaciones aparentemente contrapuestas e irreconciliables, existen intereses reales comunes a sindicatos, asociaciones empresariales y Gobierno en aras de un mayor bienestar económico y social interno y una completa incardinación en la economía internacional.

19. Sole, C., op. cits, p. 22. 